\title{
Near-IR period-luminosity relations for pulsating stars in $\omega$ Centauri (NGC 5139) (Corrigendum)
}

\author{
C. Navarrete ${ }^{1,2}$, M. Catelan ${ }^{1,2,3}$, R. Contreras Ramos ${ }^{2,1}$, J. Alonso-García ${ }^{4,2,1}$, \\ F. Gran ${ }^{1,2}$, I. Dékány ${ }^{5}$, and D. Minniti ${ }^{6,2,7}$ \\ ${ }^{1}$ Instituto de Astrofísica, Pontificia Universidad Católica de Chile, Av. Vicuña Mackenna 4860, 782-0436 Macul, Santiago, Chile \\ e-mail: cnavarre;mcatelan;rcontre@astro.puc.cl \\ 2 Instituto Milenio de Astrofísica, Santiago, Chile \\ 3 Centro de Astro-Ingeniería, Pontificia Universidad Católica de Chile, Santiago, Chile \\ 4 Unidad de Astronomía, Facultad Cs. Básicas, Universidad de Antofagasta, Av. U. de Antofagasta 02800, Antofagasta, Chile \\ 5 Astronomisches Rechen-Institut, Zentrum für Astronomie der Universität Heidelberg, Mönchhofstr. 12-14, 69120 Heidelberg, \\ Germany \\ ${ }^{6}$ Departamento de Fisica, Facultad de Ciencias Exactas, Universidad Andres Bello, Av. Fernandez Concha 700, Las Condes, \\ Santiago, Chile \\ 7 Vatican Observatory, V00120 Vatican City State, Italy
}

A\&A 604, A120 (2017), DOI: 10.1051/0004-6361/201630102

Key words. stars: variables: RR Lyrae - stars: variables: Cepheids - stars: variables: delta Scuti globular clusters: individual: NGC 5139 - infrared: stars - errata, addenda

An incorrect zero point for the empirical period-luminositymetallicity (PL-Z) relations for RR Lyrae (RRL) stars in $\omega$ Centauri (NGC 5139) was inadvertently reported in Table 2 and Eqs. (4) and (5) of our original paper. The corrected table and equations are given below. The actual analysis that is described in the paper was based on the correct zero points, and so all of the results reported in our original publication remain unchanged.

Table 2. Empirical PL-Z relations for RRL stars in $\omega$ Cen.

\begin{tabular}{ccccc}
\hline \hline Band & $a$ & $b$ & $c$ & $R^{2}$ \\
\hline$J$ & $-1.774 \pm 0.061$ & $0.153 \pm 0.027$ & $13.146 \pm 0.075$ & 0.936 \\
$K_{\mathrm{S}}$ & $-2.232 \pm 0.044$ & $0.141 \pm 0.020$ & $12.817 \pm 0.054$ & 0.985 \\
\hline
\end{tabular}

Notes. The relation has the form $m_{X}=a \log P+b[\mathrm{Fe} / \mathrm{H}]+c$, where $X$ corresponds to the bandpass.

$$
\begin{aligned}
M_{J}(\mathrm{RRL})=- & (1.77 \pm 0.06) \log P \\
& +(0.15 \pm 0.03)[\mathrm{Fe} / \mathrm{H}]-(0.56 \pm 0.08) \\
M_{K_{\mathrm{S}}}(\mathrm{RRL})=- & (2.23 \pm 0.04) \log P \\
& +(0.14 \pm 0.02)[\mathrm{Fe} / \mathrm{H}]-(0.89 \pm 0.05)
\end{aligned}
$$

Acknowledgements. We thank Vittorio Braga for bringing the error to our attention. 